\title{
LA JUDICIALIZACIÓN DE LA PROTESTA Y SUS RESULTADOS: UNA TIPOlOGía DE LA MOVILIZACIÓN LEGAL EN EL CHILE POST-TRANSICIÓN (1990-2018)
}

\author{
The Judicialization of Protest and Its Outcomes: A Typology of Legal \\ Mobilization in Post-Transitional Chile (1990-2018)
}

\author{
ANITA PERRICONE \\ Pontificia Universidad Católica de Chile
}

\begin{abstract}
RESUMEN
Existe abundante literatura sobre los resultados de la movilización legal, aunque no hay acuerdo sobre su capacidad de incidir en la toma de decisiones. Este artículo explica los resultados de distintos usos de la movilización legal mediante la exploración de los recursos organizacionales, las oportunidades políticas y legales de una serie de actores que movilizaron la ley en Chile en distintos ámbitos durante el periodo analizado. Basándose en la observación de estas variables, se identifican cuatro usos de la movilización legal: residual por parte de organizaciones medioambientales e indígenas; exclusivo por parte de los afiliados de las aseguradoras de salud privada; predominante por parte del movimiento en contra de la píldora del día después; y simbólico por parte de las minorías sexuales. Se argumenta que la movilización legal logró incidir en la toma de decisiones solo al ser utilizada de manera simbólica.
\end{abstract}

Palabras clave: movilización legal, decisiones políticas, recursos organizacionales, oportunidades políticas, oportunidades legales.

\begin{abstract}
There is abundant literature on the outcomes of legal mobilization, although there is no agreement on its effective influence on decision-making. This article explains the outcomes of different uses of legal mobilization by exploring the organizational resources, political and legal opportunities held by a series of actors who mobilized the law during the period under study. Based on the observation of these variables, four uses of legal mobilization are identified: residual by environmental organizations; exclusive by private health insurance affiliates; predominant by the anti-day after pill movement; symbolic by sexual minorities. It is argued that legal mobilization effectively influenced decision-making only when used in a symbolic way.
\end{abstract}

Keywords: legal mobilization, decision-making processes, organizational resources, political opportunities, legal opportunities. 


\section{INTRODUCCIÓN}

Por mucho tiempo, la ciencia política ha dejado de lado el análisis del sistema judicial, considerándolo como un objeto de estudio distinto a la política (Zemans 1983). Con algunas excepciones significativas (Dahl 1957; O'Donnell 2001; 2010; Hilbink 2007; Brinks 2012), el llamado a analizar el poder judicial como parte integrante del sistema político ha quedado mayormente inescuchado. Por su lado, los autores que se han abocado al estudio de la participación política (Almond y Verba 1963; Verba 1967; Verba, Nye y Kim 1978; Barnes y Kaase 1979; Verba y Nye 1987), han mirado poco o nada al sistema legal al momento de identificar los distintos canales a través de los cuales la ciudadanía puede intentar influenciar la toma de decisiones políticas.

La literatura sobre la movilización legal (ML), definida como la interpelación de los tribunales por parte de actores tanto individuales como colectivos con la finalidad de incidir en la toma de decisiones, abunda en los países anglosajones, caracterizados por sistemas de derecho común. En dichos sistemas, las decisiones de los jueces constituyen precedentes vinculantes, y las cortes son un canal de participación muy utilizado, además de un potente generador de cambios legales. Sin embargo, en ocasiones, la ML ha resultado ser un canal efectivo para influenciar a los tomadores de decisiones políticas también en países con sistemas de derecho civil. Pero ¿por qué, independientemente del resultado de los fallos, en algunos casos la ML logra incidir en la toma de decisiones, o incluso impulsar reformas legales? ¿Por qué en otros pareciera obstaculizar el cambio?

¿Por qué, en el caso chileno, minorías como los pacientes con VIH y los integrantes del Movimiento por la Liberación Homosexual (MOVILH) lograron, a través del recurso a los tribunales, la cobertura universal de la triterapia en 2001 y el Acuerdo de Unión Civil (AUC) en 2015? ¿Por qué organizaciones ambientalistas y comunidades indígenas no lograron revertir, o lo hicieron con mucha dificultad, las decisiones tomadas por la administración ambiental, como sucedió en los casos de las centrales hidroeléctricas Cuervo y Añihuerraqui? Y ¿por qué el movimiento conservador en contra de la "píldora del día después" no logró impedir la adopción de la Ley que garantiza la Anticoncepción de Emergencia (AE) en 2010, y las cientos de miles de acciones legales interpuestas, entre 2008 y la actualidad, en contra de las Instituciones de Salud Previsional (ISAPREs) no lograron promover la adopción de una reforma al sistema de salud privada?

La selección del caso chileno, cuyo sistema judicial es tristemente famoso por la falta de asertividad frente a las violaciones de derechos humanos ocurridas en dictadura (Hilbink 2007), es crucial para la evaluación de la contribución de dicho sistema al proceso de expansión de los derechos de la ciudadanía después de la transición. Dilucidar si la arena judicial constituyó un canal de participación política eficaz desde la vuelta a la democracia resulta más crucial aún de cara a los constreñimientos impuestos por la Constitución de 1980, ela- 
borada en plena dictadura. Ésta otorga mayor protección a las libertades económicas que a los derechos sociales, siendo cuestionada al punto de motivar, en abril de 2020, un plebiscito para ratificar la voluntad del pueblo chileno de reemplazarla.

Este artículo explica los resultados de distintos usos de la ML mediante la exploración de los recursos organizacionales, las oportunidades políticas y legales de actores que movilizaron la ley en Chile en distintos ámbitos durante el periodo analizado. Basándose en la observación de estas variables, se identifican cuatro usos de la ML: residual por parte de organizaciones medioambientales e indígenas; exclusivo por parte de los afiliados de las aseguradoras de salud privadas; predominante por parte del movimiento en contra de la píldora del día después; simbólico por parte de las minorías sexuales. Se argumenta que la ML logró incidir en la toma de decisiones solo al ser utilizada de manera simbólica.

\section{MARCO TEÓRICO}

La ML se convirtió en un objeto de estudio desde la emisión de la sentencia Brown vs. Board of Education de la Corte Suprema de los Estados Unidos el 17 de mayo de 1954, sobre la segregación de negros en las escuelas públicas. Si bien se desarrolló una extensa literatura sobre la ML, que se consolidó en un nuevo campo de investigación en las décadas siguientes, sus resultados siguen siendo objeto de controversia. De acuerdo con sus primeros analistas, la ML es una estrategia conservadora dominada por las élites, a través de la cual es muy poco probable cambiar el estatus quo. Scheingold (1974), por ejemplo, desconfía del "mito de los derechos", argumentando que la ML implica la pérdida de oportunidades formidables cuando es la única estrategia utilizada. De manera similar, Handler (1978) reconoce la existencia de obstáculos significativos para los cambios legalmente estimulados, mientras Rosenberg (1991) argumenta que, además de ser ineficaces, las estrategias legales absorben recursos que podrían ser empleados de otras maneras, reduciendo la probabilidad de que los actores logren sus objetivos. Aunque predominantemente escépticos, estos autores señalan que la interposición de acciones legales, en combinación con otras estrategias, puede generar efectos indirectos o no intencionales, como por ejemplo legitimar ciertas demandas, catalizar la movilización, apalancar oportunidades latentes, otorgar ventajas tácticas, concientizar a la opinión pública y defender intereses subrepresentados (Scheingold 1974; Handler 1978).

La literatura posterior mantiene una visión pesimista de los efectos directos de la ML, pero es más optimista acerca de sus efectos indirectos. Según McCann (1994), por ejemplo, la ML educa a los actores sociales, promoviendo su activismo e interés; legitima sus reclamos a través del lenguaje de los derechos; promueve la acción colectiva, aumentando las expectativas sobre el reconocimiento de sus demandas reformuladas en clave de derechos; y forja su "conciencia legal", transformando "los corazones, mentes e identidades sociales" 
de las personas (McCann 1994: 230). Smulovitz (2008), por su parte, argumenta que los actores sociales continúan usando el sistema judicial a pesar de las derrotas que puedan haber sufrido, en el entendido de que éste constituye una arena adicional de negociación y disputa política.

También hay autores que reportan las ventajas de las derrotas judiciales para los fines internos y estratégicos de los actores. Boutcher (2010) destaca el potencial de la derrota judicial en términos de recaudación de fondos, incorporación de nuevas estrategias y construcción de nuevas alianzas. NeJaime (2011) demuestra que la derrota judicial puede contribuir al fortalecimiento de la identidad organizacional y a la movilización de los constituyentes, convirtiéndose en una estrategia que permite "ganar perdiendo". Otros han examinado "la ventaja de perder" (Depoorter 2013) y el "éxito sin victoria" (Contesse y Lovera 2008), mostrando que la derrota judicial da a conocer las desgracias de individuos o grupos desfavorecidos bajo el marco legal existente, estimulando reacciones públicas, iniciativas legislativas o incluso profundas reformas. Por último, algunos sostienen que recurrir a los tribunales en condiciones desfavorables puede servir para atraer visibilidad y asistencia desde el extranjero, proporcionando una ruta hacia los tribunales internacionales (Simmons 2009; Sikkink 2011).

Sin embargo, no faltan las visiones escépticas de autores que advierten los efectos indirectos negativos de la ML. Coglianese (2001), por ejemplo, argumenta que el movimiento ambientalista estadounidense erró al adoptar la estrategia legal, dirigida a lograr objetivos acotados, en lugar de apuntar al fortalecimiento de los valores sociales subyacentes a sus demandas. Otros han señalado que, concentrándose en la estrategia legal, los movimientos sociales pueden perder su poder disruptivo y de agenda-setting (Brown-Nagin 2005) y que la mayoría de las veces la ML se desarrolla sobre estructuras institucionales que inhiben el cambio (Pieterse 2008).

Otros analistas señalan la existencia de efectos indirectos perjudiciales ligados tanto a la victoria como a la derrota judicial, así como al cuándo y al cómo de la ML. De acuerdo con Barkan (2013), la victoria judicial de un actor puede estimular la reacción de sus oponentes, o bien producir cambios irrelevantes favoreciendo el mantenimiento del estatus quo. Para otros, las derrotas judiciales son susceptibles de perjudicar a los actores sociales tanto a nivel político como organizacional, exponiéndolos a una pérdida de credibilidad y apoyo (Gloppen 2013). Yamin y Gloppen (2011) enfatizan el rol del timing de la ML postulando que, cuando es la primera medida tomada, puede jugar en contra de la formación de movimientos sociales fuertes, mientras que para Brinks y Gauri (2014) las áreas de políticas dominadas por litigios con efectos individualizados tienen más probabilidad de experimentar resultados regresivos.

Construyendo sobre la literatura revisada, que discute si la ML es una herramienta más o menos eficaz, este artículo apunta a esclarecer las circunstancias bajo las cuales la ML permite incidir en la toma de decisiones políticas a través 
de la comparación de seis contiendas sociales complejas. La hipótesis es que las condiciones estructurales bajo las cuales la ML es emprendida están directamente relacionadas con sus resultados, donde por condiciones estructurales entendemos la configuración de recursos organizacionales y oportunidades políticas y legales con las que cuentan los actores que movilizan la ley. Si bien existen estudios que analizan el rol de las oportunidades políticas y legales en la eficacia de la ML (Hilson 2002), este artículo realiza un aporte teórico al integrar los recursos organizacionales como un factor determinante para la eficacia de la misma. Además, esta propuesta constituye una contribución a la escasa literatura comparada sobre la ML, que ha sido objeto principalmente de estudios de caso.

\section{METODOLOGÍA}

La selección de los casos responde a tres criterios: el uso del recurso de protección, acción constitucional a través de la cual es posible, en un plazo de 30 días y de forma gratuita, reclamar la vulneración de algunos de los derechos fundamentales listados en el Artículo 19 de la Constitución; la invocación de los derechos a la vida (Artículo 19.1), a la igualdad ante la ley (Artículo 19.2) y a la propiedad (Artículo 19.24), los más relevantes en la litigación por medio del recurso de protección, ya que a través de éstos se suelen litigar reclamos sobre derechos que no se encuentran constitucionalmente protegidos, o lo están pero de manera incompleta (Navarro 2012); y la complejidad de las contiendas sociales en el marco de las cuales ocurrió la ML.

La vía de acceso a la justicia más utilizada por actores tanto individuales como colectivos en Chile es el recurso de protección (Navarro 2012). En el Artículo 19 , sin embargo, solo figuran como susceptibles de ser objeto de garantía constitucional una serie de derechos civiles, políticos y libertades económicas. El derecho a la salud, por ejemplo, está restringido a la posibilidad de elegir entre el Fondo Nacional de Salud (FONASA) y las Instituciones de Salud Previsional (ISAPREs).

El derecho a la propiedad es objeto de cientos de miles de recursos de protección cada año, posicionándose como el más frecuentemente resguardado por los tribunales chilenos (Navarro 2012; Allard et al. 2016). Motivo de ello son los recursos interpuestos en contra de las ISAPREs por las alzas en el precio base de los planes de salud, que al ser unilaterales violentan el derecho a la propiedad de los afiliados sobre el servicio contratado. Siguen en importancia el derecho a la igualdad, reivindicado por grupos históricamente invisibilizados por la sociedad chilena, como las minorías sexuales y los pueblos indígenas, y el derecho a la vida, que ha jugado un papel determinante en casos emblemáticos en el ámbito de la salud, como aquellos de la píldora del día después y de la triterapia.

El tercer criterio de selección, finalmente, alude a la complejidad que caracteriza los seis episodios analizados, medida en términos de la heterogeneidad 
de los actores sociales e institucionales que jugaron un papel en éstos, y de su larga duración: los casos involucraron a actores de la sociedad civil, tanto individuales como colectivos; a figuras políticas a nivel local, regional y nacional; a distintos eslabones de la administración del estado y del sistema judicial. Además, todos se extendieron por entre 5 y 10 años desde la formulación de la demanda que originó el recurso a los tribunales.

Con la finalidad de complementar la información recolectada a partir de fuentes primarias y secundarias, entre mayo de 2015 y octubre de 2017 se realizaron 33 entrevistas en profundidad ${ }^{1}$ con actores involucrados en los seis episodios, en particular a los litigantes, sus abogados y a expertos. En el caso de las comunidades indígenas se realizó observación participante ${ }^{2}$ en tres instancias, dada la imposibilidad de acceder a la información sin desarrollar previamente cierto vínculo de confianza. Para el análisis de la información se utilizan las narrativas analíticas (NA), un enfoque que se apoya en la teoría de juegos, pero que no es estrictamente deductivo. Las NA resaltan ciertas características de la estructura, con la finalidad de explicar cómo éstas moldean las decisiones individuales y los resultados colectivos, tomando en cuenta preferencias, incentivos y objetivos de los actores involucrados en el afán de identificar mecanismos causales (Levi 2006). La principal fortaleza de las NA es la creación de modelos teóricos que ayudan a esclarecer los mecanismos y resultados alcanzados por los actores en distintos contextos. Su mayor debilidad, en cambio, es la dificultad para generalizar a partir de éstas: las NA requieren de un conocimiento profundo de los casos seleccionados, y por lo mismo no pretenden formular teorías universales, sino dar cuenta de la especificidad de los fenómenos sociales observados. Esto no significa que no sea posible y deseable aspirar a generalizaciones de las conexiones existentes entre la estructura y los agentes (Levi 2006), como se tratará de hacer aquí.

\section{ANÁLISIS DE CASOS EMPÍRICOS}

Para el análisis de los casos empíricos se tornará la mirada hacia los recursos organizacionales y oportunidades políticas y legales que los actores que protagonizaron los seis episodios seleccionados poseían, los que serán catalogados como abundantes o escasos. Si bien los teóricos de la movilización de recursos mencionan la existencia de recursos internos y externos (McCarthy y Zald 1977; Morris 1981), aquí se propone una distinción entre recursos materiales y activistas. Dicha distinción es importante porque podrían existir desajustes. Por recursos materiales se entenderán los recursos económicos y humanos a disposición de un actor, por ejemplo la existencia de liderazgos y/o equipos

De las 33 entrevistas realizadas, en este artículo, que es el resumen del argumento de mi tesis doctoral defendida en diciembre de 2017, se utilizaron solamente algunas. Las entrevistas se identifican con un numero precedido por el signo \#.

2 Las citas derivadas de las instancias de observación participante se identifican en el texto con un número precedido por el signo \#OP. 
conformados por profesionales, entre ellos abogados, y/o el apoyo brindado por estructuras de soporte legal (Epp 1998), tales como ONGs o abogados de causa que ofrecen servicios legales gratuitos. Se entenderá que un actor dispone de recursos materiales abundantes si es capaz de financiar una estrategia legal y/o una campaña pública. Por recursos activistas se entenderá la capacidad de un actor social de movilizar personas más allá de sus miembros, como los adherentes a una determinada causa y/o sus potenciales beneficiarios. Se entenderá que un actor cuenta con recursos activistas abundantes si logra formar coaliciones de alcance nacional o internacional, y si enmarca su demanda de manera incluyente, logrando maximizar los adherentes a su causa, como lo hizo el MOVILH al desvincular su demanda de la unión entre personas del mismo sexo, e incluyendo a las parejas heterosexuales no casadas.

El concepto de estructura de oportunidades políticas indica el grado en que los grupos tienen la capacidad de ganar acceso al sistema político (Eisinger 1973: 25). Dado que las oportunidades políticas pueden estar distribuidas de manera desigual dentro del mismo sistema político (Tarrow 1998; Hilson 2002; Meyer y Minkoff 2004), y que los estados están insertos en el sistema internacional, las definiciones clásicas deberían ser enriquecidas por elementos subnacionales (Amenta et al. 2002) e internacionales (Van der Heijden 2006). Se analizarán dos grandes rasgos del sistema político: su apertura y su receptividad. Se considerará abierto aquel ámbito caracterizado por burocracias y leyes permeables a, o que cumplen con, los estándares internacionales, y/o que garantizan mecanismos de participación ciudadana eficaces (Amenta et al. 2002). Se considerará receptivo aquel ámbito en el que los actores sociales cuentan con aliados, ya sea domésticos o internacionales, en relación a sus demandas. Como aliados domésticos relevantes serán considerados los miembros del poder ejecutivo, cuyos compromisos electorales y preferencias de política pública favorecen el logro de una determinada demanda. Entre los aliados políticos internacionales se considerará la existencia de actores internacionales que promuevan una determinada causa, como el Programa Conjunto de las Naciones Unidas sobre el VIH/Sida (ONUSIDA), y el movimiento global de Lesbianas, Gays, Bisexuales, Transexuales e Intersexuales (LGBTI).

Diversos estudiosos de la ML han reivindicado el carácter autónomo de las oportunidades y amenazas que pueden residir en el sistema judicial (Hilson 2002; Andersen 2005; Vanhala 2011). Dado que, como sus contrapartes políticas, las oportunidades legales tienen tanto componentes estructurales como contingentes, se analizarán dos atributos del sistema judicial: su apertura y su receptividad. Un sistema judicial será considerado abierto si no existen costos para el acceso efectivo a la justicia. Por ejemplo, aunque una acción legal no tenga costos asociados, como en el caso del recurso de protección, cuando se trata de demostrar la afectación del medioambiente es necesario encargar estudios que, a menudo, solo las empresas proponentes, y no las comunidades afectadas, pueden pagar, configurando un acceso desigual a la justicia. 
El sistema judicial se considerará receptivo toda vez que los argumentos contenidos en la acción legal han sido avalados por los jueces en pasado. La receptividad de los tribunales puede variar de acuerdo a su composición, ya que los jueces pueden tener distintas actitudes y preferencias hacia los otros poderes del estado (Tate and Vallinder 1995). Por ejemplo, entre los años 2011 y 2013, la Corte Suprema abandonó la "deferencia técnica", mantenida durante más de una década hacia la administración ambiental, en favor de una mayor "activismo judicial", lo cual se materializó en el rechazo de una serie de Estudios de Impacto Ambiental de megaproyectos extractivos y energéticos por razones más técnicas que jurídicas (García y Verdugo 2013). Las preferencias y actitudes de los jueces chilenos también dependen de su pertenencia a distintos eslabones del sistema judicial, siendo los jueces de las Cortes de Apelaciones menos conservadores que aquellos de la Corte Suprema y del Tribunal Constitucional (Muñoz 2014).

A continuación, la Tabla 1 presenta cuatro tipos de ML, establecidos de manera deductiva de acuerdo a las configuraciones de recursos y oportunidades que las caracterizan.

Tabla 1. Los tipos de ML según recursos y oportunidades de los actores

\begin{tabular}{lcccc}
\hline & Residual & Exclusivo & Predominante & Simbólico \\
\hline Recursos organizacionales & Escasos & Escasos & Abundantes & Abundantes \\
Oportunidades políticas & Escasas & Escasas & Escasas & Abundantes \\
Oportunidades legales & Escasas & Abundantes & Abundantes & Escasas \\
\hline
\end{tabular}

\section{La ML Residual}

A continuación, se analizan dos episodios ocurridos en el ámbito del Sistema de Evaluación Ambiental (SEA). El primer episodio involucró a cuatro comunidades mapuche que se opusieron a la construcción de la hidroeléctrica de pasada Añihuerraqui en territorio indígena (Región de la Araucanía). Dichas comunidades contaban con recursos materiales escasos, aunque recibieron la asesoría legal gratuita del Observatorio Ciudadano, ONG para la defensa de los derechos de los pueblos indígenas basada en Temuco, lo que les permitió desplegar una campaña legal que persistió durante varios años (\#OP3). Las comunidades contaban, además, con recursos activistas escasos, no logrando construir una coalición de alcance nacional, lo que junto a un enmarcamiento de la demanda centrado en la defensa del agua y de los territorios dificultó que la causa alcanzara audiencias más allá de la zona sur del país (\#OP1; \#OP3).

A pesar de la apertura política que significó la ratificación del Convenio 169 de la Organización Internacional del Trabajo (OIT) en 2008, cuya piedra angular 
es el derecho a la consulta indígena (Artículo 6), la regulación de éste por medio de dos reglamentos (DS 124 y DS 66) no logró garantizar el cumplimiento en buena fe de los estándares internacionales por parte de las autoridades chilenas (\#2; \#24). Un proceso de consulta tuvo lugar entre 2013 y 2015 con la finalidad de alcanzar un acuerdo entre las partes. La consulta, sin embargo, fue boicoteada y por tanto invalidada por diversas comunidades, las mismas que interpusieron las acciones legales, decididas a no entregar acceso a su territorio a la empresa (\#16).

En cuanto a la receptividad del sistema político, la demanda de las comunidades enfrentaba un escenario adverso, debido a la reciente decisión del entonces Ministro de Energía Máximo Pacheco de renovar la matriz energética del país fomentando la instalación de pequeñas centrales hidroeléctricas de pasada ${ }^{3}$ en vez que grandes represas, particularmente impopulares tras el éxito de la campaña internacional Patagonia Sin Represas, finalizada a impedir la construcción de una serie de represas en la Patagonia chilena. A pesar de que las comunidades contaran con el apoyo de la Corporación Nacional de Desarrollo Indígena (CONADI), la Comisión de Evaluación Ambiental (CEA) de la Araucanía dio luz verde al proyecto en julio de $2015^{4}$.

Estimando que no se había respetado su voluntad durante el proceso de consulta, las comunidades interpusieron un recurso de protección invocando el derecho a la igualdad ante la ley (Artículo 19.2). Si bien las comunidades no incurrieron en costos extra para acceder a la justicia, se enfrentaron a un escenario adverso en tribunales, caracterizado por el rechazo de una serie de recursos interpuestos en contra de la aprobación de otros proyectos energéticos en la Araucanía ${ }^{5}$. La Corte de Apelaciones de Temuco ${ }^{6}$ y la Corte Suprema7 ratificaron la vigencia de la Resolución de Calificación Ambiental otorgada a la hidroeléctrica, desestimando el hecho que la consulta requiere alcanzar un acuerdo. Aunque la empresa ya se encontrara en proceso de instalación, las comunidades afectadas impugnaron las concesiones eléctricas concedidas a la misma e interpusieron dos ulteriores recursos en el intento de impedir o retrasar su construcción. Sin embargo, dichas acciones legales fueron rechazadas por la Corte Suprema en agosto de $2018^{8}$.

El segundo caso se relaciona con la construcción de la represa Cuervo en la región de Aysén, que había sido objeto de la campaña Patagonia Sin Represas. Lanzada por el Consejo de Defensa de la Patagonia en 2007, esta campaña contó con la membresía de más de 80 organizaciones nacionales e internacionales, y

Oficio N 145/2016 del 27 de enero de 2016, de la Comisión de Medio Ambiente y Recursos Naturales de la Cámara de Diputados de la República.

Resolución Nº 177 de la Comisión de Evaluación Ambiental de la Araucanía del 20-07-2015.

Doña Alicia en Curacautín, Los Aromos en Pitrufquén, y la línea de transmisión Carilafquén-Malalcahuello en Melipeuco.

Causa rol N 3956 de 2015, Corte de Apelaciones de Temuco, del 16 de noviembre de 2015.

Fallo N ${ }^{\circ} 33.154-2015$ de la Tercera sala de la Corte Suprema, del 29 de diciembre de 2015.

Causa rol 23.143-2018 y Causa rol 23.272-2018 de la Corte Suprema. 
fue beneficiada por los abundantes recursos financieros inyectados en ella por el empresario y filántropo Douglas Tompkins. Sin embargo, una vez rechazado el emblemático proyecto HidroAysén en junio de 2014, la campaña finalizó y el proyecto Cuervo se aprobó apenas un mes más tarde 9 . Sin el respaldo de la campaña, las comunidades y organizaciones medioambientales locales que se oponían al proyecto, basadas en Coyhaique y Puerto Aysén, y desprovistas de recursos activistas más allá del nivel local (\#8), interpusieron un recurso de protección con el apoyo de la ONG Fiscalía del Medioambiente (FIMA), el que concluyó en un fallo desfavorable de la Corte Suprema ${ }^{10}$.

En cuanto a la apertura del sistema político, el Sistema de Evaluación Ambiental se caracteriza por la debilidad de los procesos de participación ciudadana, por un sesgo a favor de las empresas en desmedro de las comunidades y del nivel central a costas del regional. Después del rechazo de HidroAysén, además, el gobierno necesitaba enviar el mensaje de que no todos los grandes proyectos de inversión serían rechazados sistemáticamente (\#5). En otras palabras, los consensos políticos favorecían la aprobación del proyecto, lo que junto a lo mencionado anteriormente configuraba un escenario de oportunidades políticas escasas.

En cuanto a apertura del sistema legal, el acceso efectivo a la justicia constituyó un desafío. Dados los riesgos que representaba la construcción de una represa en una zona altamente sísmica, a la empresa proponente se le pidieron estudios de suelo, de agua y vulcanológicos como parte del Estudio de Impacto Ambiental. FIMA, por su parte, tuvo que realizar estudios con los recursos humanos a disposición, lo que sin financiamiento no logró los objetivos esperados (\#11). La central Cuervo fue aprobada en julio de 2014 por la Comisión de Evaluación Ambiental de Coyhaique, tras lo cual organizaciones ambientalistas locales y ex miembros del Consejo de Defensa de la Patagonia interpusieron un recurso de protección, cuyo rechazo por parte de la Corte Suprema determinó que el caso alcanzara el Tribunal Ambiental de Valdivia, que finalmente anuló la Resolución de Calificación Ambiental del proyecto en enero de 2018 ${ }^{11}$, aunque la empresa ya hubiera renunciado a la realización del mismo.

En el ámbito medioambiental, el recurso a los tribunales constituyó una estrategia residual, emprendida tras el fracaso o la imposibilidad de emprender estrategias alternativas como la protesta y el lobby político. Enfrentados a una institucionalidad impermeable y a consensos políticos adversos, los actores visualizaron como opción residual la interposición de ulteriores acciones legales. En su versión residual la ML fue utilizada para intentar revertir, o retrasar las consecuencias de decisiones tomadas por instancias administrativas. La multiplicación de las acciones legales permitió que los opositores de las represas ganaran tiempo, en la esperanza de que las condiciones que determinan la via- 
bilidad económica y social de los proyectos variaran y fueran reevaluadas por las empresas proponentes, otorgándoles una ventaja táctica.

El análisis de los casos en el ámbito medioambiental alumbra un panorama sombrío tanto para activistas pro medioambiente como para comunidades indígenas, al menos hasta 2017. Sin embargo, a seis años de la entrada en funcionamiento de los Tribunales Ambientales, su jurisprudencia deja entrever nuevas luces en cuanto a la aplicación de principios del derecho internacional del medio ambiente, como el principio precautorio aplicado en el caso de la central Cuervo, entre otros, sugiriendo que incluso un recurso residual a la ML puede arrojar un resultado favorable para los litigantes, premiando su insistencia en la interposición de acciones legales.

\section{La ML exclusiva}

Desde 2008, la interposición de recursos de protección por parte de los afiliados de las ISAPREs en contra de las alzas anuales y unilaterales del precio de los planes ha crecido exponencialmente, llegando a un máximo de 185.000 en 2016. Estos recursos pueden ser considerados como actos individuales de protesta por las alzas ilegales, y como el emblema de la necesidad de reformar el sector de la salud privada en su conjunto, agudizada por la falta de una alternativa pública de calidad.

Sin embargo, no existe ningún movimiento ni organización que aglutine las demandas de reforma a las ISAPREs de manera permanente. El intento de formar la Corporación de Usuarios de ISAPREs (CORPUSAPREs) en 2000, con la finalidad de representar a los afiliados y brindarles apoyo legal, no prosperó debido al individualismo que caracteriza a la clientela de estas empresas, relacionado con la competitividad introducida por la revolución cultural impulsada por el régimen militar, y seguida por una fuerte caída de los índices de asociatividad (\#28). De haber prosperado, esta organización pudo haberse convertido en una promotora de la reforma al sistema de salud privado. En su defecto, el descontento social en este ámbito ha sido aprovechado como una oportunidad de negocio por parte de cientos de servicios legales en línea que ofrecen la interposición gratuita de recursos de protección en contra de las ISAPREs. Tras la masificación de estas acciones legales y la cobertura brindada por los medios de comunicación, la demanda por la reforma del sistema de salud privado se instaló en la agenda política, aunque no logró concretarse (\#32).

En las últimas dos administraciones (Piñera 2010-2014 y Bachelet 2014-2018) faltó consenso político para llevar a cabo una reforma, que fue objeto de dos Comisiones Parlamentarias durante el primer período de gobierno. Dichas comisiones propusieron una reforma integral del sistema de salud (Ley Larga de ISAPREs) y la creación de un índice de precios al consumidor en el sector de la salud, entre otras propuestas (Ley Corta de ISAPREs). Sin embargo, ninguna de las dos prosperó a raíz de la conservación de algunos criterios de selección 
del riesgo que la izquierda no estuvo dispuesta a mantener (\#22). Una tercera Comisión fue instituida en 2014 por la coalición de izquierda Nueva Mayoría, que a diferencia de su antecesora, la Concertación de Partidos por la Democracia, incluyó al Partido Comunista. El desplazamiento del eje político hacia la izquierda, y la propuesta de creación de un fondo común que acabaría con las ISAPREs, terminó por impedir nuevamente alcanzar un consenso para reformar el sistema de salud, esta vez por la radicalidad de la propuesta (\#25).

En cuanto a apertura del sistema legal, los litigantes encontraron una situación favorable. La impugnación de las alzas ocurre mediante la interposición de recursos de protección para reestablecer la garantía del derecho a la propiedad (Artículo 19.24), una de las piedras angulares de la Constitución, que implica la ilegalidad de modificar las condiciones contractuales de manera unilateral. Dado que los recursos de protección no sientan ningún precedente, los tribunales han fallado sistemáticamente a favor de los litigantes en cientos de miles de casos similares. Además de ser gratuita, la interposición de un recurso garantiza al litigante el reembolso de los montos cobrados ilegalmente a raíz de las alzas unilaterales. Para los abogados, la interposición de recursos de protección representa una ganancia económica equivalente al valor de los costos del juicio, pagadas por la ISAPRE recurrida, menos el valor del reembolso al litigante, por lo cual ha ocurrido incluso que los abogados le pagaran a las víctimas de las alzas por contratar su servicio (\#28).

En el ámbito de la salud privada, por el carácter individual y costoso de las prestaciones, priman la escasez de recursos y de oportunidades políticas y, dada la ausencia del derecho a la salud en el ámbito de garantía del recurso, también escasean las oportunidades legales. El caso analizado es más bien una excepción, ya que la norma invocada es el derecho a la propiedad. En el caso de las ISAPREs, la ML es la única estrategia emprendida por los afectados por las alzas unilaterales operadas por las empresas aseguradoras. El éxito sistemático de los casos en los tribunales, sin embargo, terminó desincentivando ulteriormente la asociatividad, ya característica del ámbito de la salud privada, silenciando la demanda por una mayor regulación del sistema. De esta forma, la ML dotó éste último de en una válvula de escape para el malestar que, en vez de acumularse y estimular la articulación de un movimiento masivo pro reforma, contribuyó a mantener el estatus quo.

\section{La ML predominante}

Otro episodio de ML en el ámbito de la salud fue protagonizado por el movimiento conservador que surgió en contra de la "píldora del día después" a comienzo de los 2000. Este movimiento, liderado por activistas y políticos "pro vida", vio el despliegue de una estrategia legal que duró casi una década (2001-2010), orientada a frenar la política de la anticoncepción de emergencia (AE). En ámbito organizacional, los actores que protagonizaron la "cruzada 
conservadora" (Muñoz 2014) para el retiro de la píldora del mercado nacional contaban con recursos materiales y activista abundantes, lo que les permitió armar, además de una campaña legal, una agresiva campaña de intimidación orientada a los laboratorios farmacéuticos que importaban el medicamento, y a las cadenas de farmacias que lo comercializaban (Casas 2008).

La campaña legal fue lanzada por la organización juvenil AGES, fundada por el otrora estudiante de derecho Juan Enrique Jara, y comenzó por impugnar la resolución del Instituto de Salud Pública (ISP) ${ }^{12}$ que regulaba la distribución de la primera marca de la píldora (Postinal) comercializada en el país. La campaña pública fue promovida por la Asociación Nacional de Consumidores (ACONOR), creada por el mismo Jara, y consistió de amenazas judiciales a laboratorios y farmacias por vender un medicamento supuestamente abortivo, y por lo tanto ilegal. Éstos últimos reaccionaron retirando la píldora del mercado hasta casi desabastecerlo, lo que motivó el inicio de un movimiento en defensa de la píldora, que integraba mujeres y profesionales de la salud (Casas 2008). A pesar de la existencia de aliados políticos influyentes, entre ellos la Iglesia Católica, los alcaldes de algunas de las comunas más grandes del país (La Florida, Puente Alto, Concepción, entre otras), y una treintena de parlamentarios que se fueron sumando a la campaña, desde el punto de vista político la situación que enfrentaba el movimiento conservador era adversa. El entonces pesidente Ricardo Lagos (2000-2006) y la otrora ministra de Salud Michelle Bachelet estaban fuertemente comprometidos con la píldora como una medida para reducir el embarazo adolescente. Además de aprobar el registro de una píldora equivalente (Postinor-2) ${ }^{13}$ el Ministerio de Salud (MINSAL) impuso multas a las cadenas de farmacias que, intimidadas por las amenazas judiciales, habían retirado los medicamentos para la $\mathrm{AE}$ de sus estanterías. Cuando los laboratorios dejaron de importar el medicamento, el Ministerio adquirió 30.000 dosis de la droga a través de la Central Nacional de Abastecimiento (CENABAST), y posteriormente obligó a las farmacias a reabastecerse y asegurar su venta (Casas, 2008).

En cuanto a oportunidades legales la situación era ambigua. Cuando el ISP autorizó la comercialización de Postinal, distintos actores interpusieron una serie de recursos de protección invocando el derecho a la vida del no nacido (Artículo 19.1). Estos fueron desestimados por la Corte de Apelaciones de Santiago por falta de legitimación activa de los presuntos afectados, aunque posteriormente la Corte Suprema respaldó el derecho a la vida del no nacido, dejando sin efecto la resolución del ISP. Cuando, en 2006, éste último aprobó las Normas Nacionales sobre Regulación de la Fecundidad ${ }^{14}$ y reiteró la legalidad de la píldora, los opositores desplegaron una estrategia legal fragmentada: la normativa fue impugnada por ciudadanos desorganizados y alcaldes opositores median- 
te recursos de protección, mientras que 31 parlamentarios de la ultraderecha conservadora interpelaron el Tribunal Constitucional (TC). La interpelación resultó exitosa, culminando en un fallo que declaró la inconstitucionalidad de la píldora en $2008^{15}$. Sin embargo, dicho fallo desencadenó protestas masivas por parte de los sostenedores de la píldora, que respaldaron la decisión del gobierno de Michelle Bachelet (2006-2010) de incumplir el mismo y legislar para garantizar la distribución de la píldora a nivel nacional (Ley 20.418 de 2010).

De manera similar a lo ocurrido en el caso de las ISAPREs, los opositores de la píldora optaron por el litigio antes de emprender otro tipo de acciones, y volvieron a enfocarse en la estrategia legal al verse enfrentados con oportunidades políticas escasas. El compromiso del gobierno con la AE era tan fuerte que, frente a la serie de fallos judiciales contrarios a la píldora, éste ejerció "resistencia legal" (Muñoz 2014) desobedeciendo el fallo del TC, lo que significó la derrota del movimiento pro-vida. Dicha derrota en la "batalla" en contra de la AE no les impidió a los conservadores continuar en "guerra" por la defensa de la vida del no nacido (\#18). Sin embargo, ello significó lidiar con los cambios que se venían configurando a nivel político y social, lo que a su vez requirió de la de-radicalización de las demandas de los grupos pro vida, obligándolos a desplazar el foco en el incipiente debate sobre el aborto (\#18).

\section{La ML simbólica}

El primer caso a analizar es la lucha de las personas con VIH por el acceso universal a la triterapia. Entre 1999 y 2001, este grupo altamente organizado desde antes de la transición a la democracia interpuso una serie de recursos de protección, alegando que la negación del acceso universal y gratuito al tratamiento antirretroviral por parte del gobierno constituía una violación del derecho a la vida (Artículo 19.1) de las personas con VIH. La interposición de los recursos fue promovida por VIVOPOSITIVO, una red de organizaciones de afectados por el VIH de alcance nacional orientada a fortalecer el discurso de los derechos en seno a un movimiento que reunía a personas altamente vulnerables en el marco legal de la época.

Las oportunidades políticas que enfrentaba inicialmente este movimiento, dado el modelo de estado subsidiario instalado durante el régimen militar, eran escasas. La idea de Jaime Guzmán, el principal ideólogo de la Constitución de 1980, era que la libertad económica fuera el motor de la economía y que el Estado se hiciera cargo de lo que excedía la capacidad de los privados. La decisión de excluir a los derechos sociales del ámbito de tutela del recurso de protección respondió a la convicción que dichos derechos "positivos", dependiendo de la capacidad económica del estado, debían quedar fuera de la garantía constitucional. Sin embargo, la creación de ONUSIDA como el principal referente de la 
lucha global en contra de la epidemia en 1996 determinó la apertura de oportunidades políticas de relevancia crucial. En 1997, VIVOPOSITIVO se posicionó como intermediario en las negociaciones entre el gobierno y ONUSIDA, incrementando de manera sustancial las probabilidades de conseguir su demanda.

La estrategia legal, por su parte, no dio los frutos esperados: los tres primeros recursos de protección interpuestos por personas con VIH en 1999 fueron declarados inadmisibles porque invocaban el derecho a la vida (Artículo 19.1). Sin embargo, de acuerdo con los jueces, se habría tratado de la vulneración del derecho a la salud (Articulo 19.9) que, no estando garantizado constitucionalmente, liberaba al Estado de toda responsabilidad. A raíz del agotamiento de los recursos internos, estos tres casos fueron elevados al Sistema Interamericano de Derechos Humanos (SIDH). Sin embargo, tras el fallecimiento de dos de los tres litigantes que estaban a la espera del fallo, la condena a muerte que la escasez de oportunidades políticas y legales significaba para las personas que vivían con VIH en el país conmocionó a la opinión pública, generando un intenso debate que tuvo el efecto de legitimar su demanda. Fruto de las presiones ejercidas por la opinión pública, VIVOPOSITIVO consiguió que el gobierno peticionara el Fondo Global de Naciones Unidas para recibir asistencia en el diseño de una política pública que permitiera el logro gradual de la cobertura del tratamiento antirretroviral (Contesse y Lovera, 2008). Luego de conseguir financiamiento internacional, VIVOPOSITIVO asesoró el gobierno en la elaboración de un proyecto de ley que garantizara la cobertura universal de algunas enfermedades catastróficas, entre ellas el VIH (Ley 19.779 de 2001).

Otra contienda social compleja en la que es posible identificar esta configuración de variables es la del Acuerdo de Unión Civil (AUC), fruto de una larga serie de acciones públicas, legales y políticas por parte de organizaciones del movimiento LGBTI, entre las que destaca el MOVILH. Desde 1990, éste sumó un número creciente de miembros, alcanzando una cantidad considerable de recursos materiales, entre los que destacan la existencia de sedes regionales, un staff permanente, liderazgos profesionales, y recursos activistas. Estos últimos hicieron posible el despliegue de una multiplicidad de estrategias a lo largo del tiempo: marchas masivas, campañas públicas, iniciativas culturales, negociaciones políticas, entre otras (\#30). Los recursos organizacionales permitieron el ejercicio de presiones constantes sobre los sucesivos gobiernos para el conseguimiento de demandas relacionadas con la diversidad sexual. El momento más álgido de las manifestaciones siguió a la adopción de una estrategia de enmarcamiento amplio de la demanda, que incluyó como beneficiarias del AUC a las parejas heterosexuales no casadas (\#30).

Si bien en los años noventa y primera mitad de la década siguiente las oportunidades políticas que enfrentaba la diversidad sexual eran muy escasas, la situación fue mejorando conforme al crecimiento del movimiento a nivel internacional. La campaña presidencial de 2009 marcó un antes y un después, al ser las uniones homosexuales incorporadas en los programas electorales de todos los candidatos. En su mensaje presidencial del 21 de mayo de 2010, el 
presidente Sebastián Piñera, quien representaba el ala liberal de la coalición de centro-derecha Alianza por Chile, se refirió a la necesidad de contar con una legislación que no discriminara a las personas por su orientación sexual. Si bien estas declaraciones pudieron haber implicado un apoyo más verbal y simbólico que relacionado con un avance legislativo efectivo, fueron sin duda síntoma de un clima político mucho menos hostil a la diversidad sexual.

La estrategia legal consistió de la interposición de una serie de recursos de protección patrocinados por el MOVILH, que apuntaban a reestablecer el derecho a la igualdad frente a la ley (Artículo 19.2) tras la negativa del Registro Civil de casar a una pareja del mismo sexo, y de legalizar en Chile dos matrimonios celebrados en el extranjero. Los recursos desafiaron la constitucionalidad del Artículo 102 del Código Civil, que contempla una definición de matrimonio limitada a la unión entre un hombre y una mujer, pero fueron fallados en contra de los litigantes por la Corte de Apelaciones de Santiago, que se limitó a aplicar la ley vigente. Sorpresivamente, sin embargo, el caso dio pie a la interpelación, por parte de un juez de esta última corte, del TC que, a pesar de desechar la presunta inconstitucionalidad del Artículo 102, emitió un fallo cuyos votos disidentes invitaban al Poder Legislativo a tomar voz en el asunto. Tras este llamado, y dadas las fuertes presiones sociales, tal como ocurrió en el caso de las personas con VIH, el Legislativo invitó a representantes del movimiento LGBTI a asesorarlo en la elaboración de una ley que regularía los derechos de las parejas homosexuales y heterosexuales no casadas (Ley 20.830 de 2015).

Este tipo de ML ocurrió en presencia de recursos organizacionales abundantes, oportunidades políticas abundantes, aunque latentes, y oportunidades legales escasas. Dado esto último, el litigio debiese haber sido considerado una opción riesgosa, ya que bien podría haber implicado una pérdida de tiempo y/o de recursos económicos. Este riesgo, sin embargo, fue enfrentado exitosamente por actores sociales que contaban con recursos materiales y activistas, y que fueron capaces de generar un debate público transversal en reacción a fallos desfavorables. Se destaca la importancia de la coexistencia de campañas públicas y legales, de una estrategia de enmarcamiento de las demandas incluyente, y de coaliciones de alcance nacional para el logro de los resultados políticos deseados.

El carácter simbólico de la ML residió en la capacidad de la derrota judicial de crear conmoción y consciencia de una causa perdida, tanto en ámbito judicial como político, y de transformarla en una oportunidad para revestir de legitimidad pública el actor y la demanda en cuestión. Si bien los protagonistas de los episodios relatados no gozaron de apoyo político mayoritario desde un principio, lo obtuvieron una vez sensibilizada la opinión pública entorno a la discriminación que motivó las acciones legales, mediante un amplio debate público. Una vez legitimadas, estas demandas se convirtieron en asuntos que requerían ser abordados de manera urgente, y cuya satisfacción por parte del gobierno de turno se tornó políticamente atractiva, a pesar de los costos económicos que 
implicó en el caso de la triterapia, y de los conflictos valóricos que acarreó dentro de la coalición de gobierno en el caso del AUC. A continuación, el Gráfico 1 representa la mecánica del argumento expuesto en este trabajo, poniendo el énfasis en las condiciones bajo las cuales cada ocurrió cada uso de la ML, los mecanismos causales que intervinieron y los resultados que obtuvieron.

Gráfico 1. Resumen del argumento

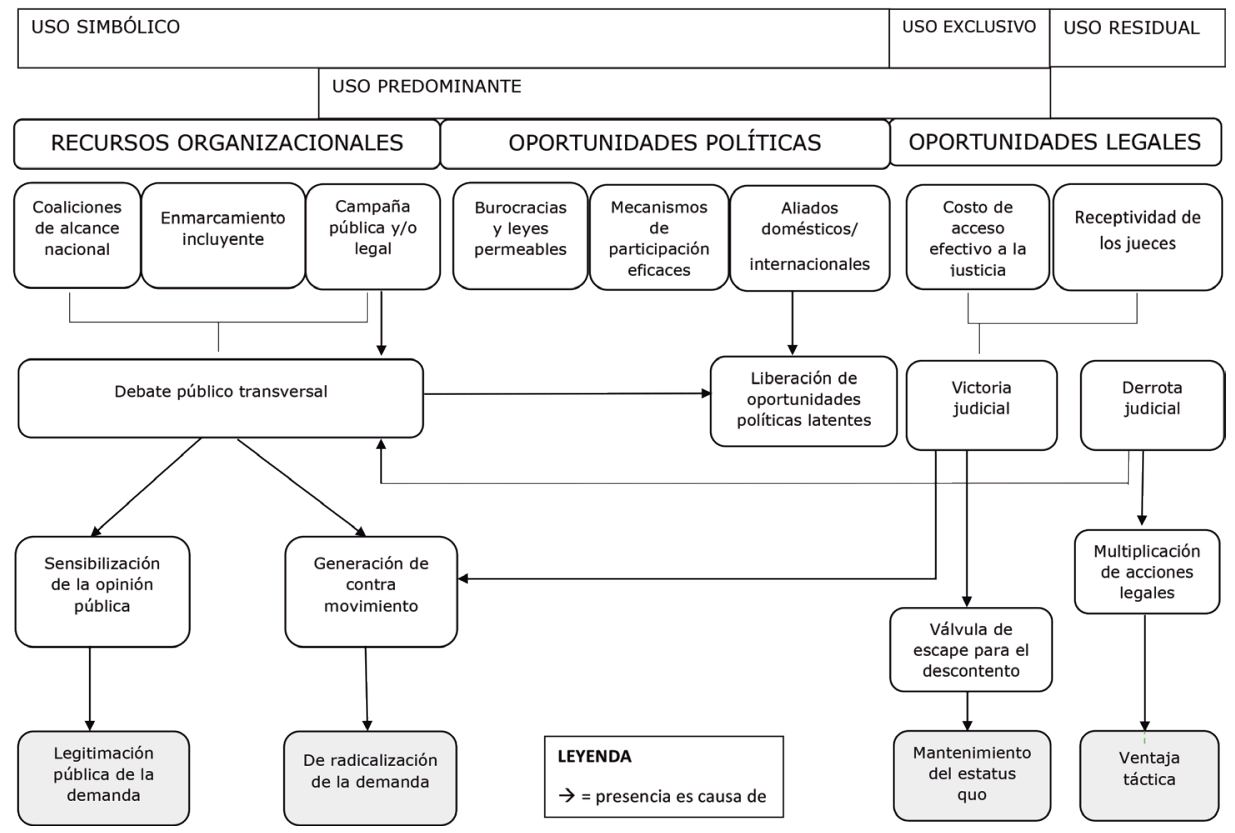

\section{CONCLUSIONES}

La conclusión principal de este artículo es que la existencia de recursos organizacionales abundantes fue una condición necesaria para el éxito de la ML en Chile durante el período analizado. Solo en presencia de campañas públicas y legales, un enmarcamiento incluyente de las demandas, y coaliciones de alcance nacional, distintos actores, aunque minoritarios, lograron ejercer influencia política a través de la ML. Se destaca la importancia de la presencia conjunta de estos factores, ya que, como demuestra el caso de la píldora, la sola existencia de campañas públicas y legales no fue capaz de garantizar el cambio, ni siquiera en presencia de una victoria judicial. Paradójicamente, los episodios en los que la ML fue más eficaz fueron aquellos en los que minorías desprovistas 
de oportunidades legales hicieron un uso simbólico del recurso de protección poniendo la discriminación que los afectaba al centro de la controversia, lo que operó como una palanca para liberar oportunidades políticas latentes.

Recursos organizacionales abundantes, sin embargo, constituyeron una condición necesaria pero no suficiente para lograr el éxito de la ML: como demuestran los episodios de VIVOPOSITIVO y del MOVILH, la presencia de oportunidades políticas, como la existencia de aliados domésticos o internacionales, jugó un rol clave en la determinación de la eficacia de las acciones legales. Así también lo demuestra el caso del movimiento pro vida, que si bien contaba con recursos abundantes, no pudo contra la voluntad férrea de los gobiernos de Lagos y Bachelet de garantizar la píldora del día después. También lo demuestra el caso del megaproyecto hidroeléctrico HidroAysén que, a pesar de no formar parte de la muestra, fue rechazado solo meses después de la toma de mando de Bachelet, cuyo segundo gobierno aprovechó de apartarse de la postura piñerista adjudicándose la reversión de una decisión altamente impopular.

Cabe preguntarse si estas conclusiones son generalizables a otros sistemas de derecho civil, donde las sentencias judiciales, a diferencia de lo que ocurre en los países anglosajones, por lo general no crean precedentes vinculantes. La diferencia entre sistemas de derecho común y civil es bastante borrosa, siendo que existen diferentes grados de valoración de la jurisprudencia de los tribunales superiores en los países de derecho civil (Vaccario 2015). Tal vez el hecho que el Código Civil establezca que solo el Legislativo está legitimado para interpretar la ley de manera vinculante, y que las sentencias tengan vigencia solo en casos puntuales, haga de Chile un caso bastante particular. Esta norma es un legado de la década de 1830, cuando la élite fundadora decidió excluir a los jueces de las decisiones políticas, cuya consecuencia fue la tendencia a canalizar las demandas sociales a través del Legislativo (Hilbink 2007). Esto último es coherente con las conclusiones de este trabajo, que ha establecido la necesidad y suficiencia de recursos organizacionales y oportunidades políticas abundantes para el éxito de la ML durante el periodo estudiado.

Finalmente, el análisis evidencia algunas características del sistema judicial chileno, y deja entrever cambios importantes ocurridos desde el retorno a la democracia. En el ámbito del medio ambiente se observó la prevalencia de una actitud deferente hacia el poder político, con la excepción de un mayor activismo entre 2011 y 2013, tras lo cual fueron establecidos y empezaron a funcionar los Tribunales Ambientales. Estos últimos han dado mayor aplicación a los principios del derecho internacional en el control jurisdiccional de la actividad administrativa (Irarrázabal y Luengo 2018) y en la implementación del derecho a la consulta indígena (Guerra 2017).

En el ámbito de la salud, los tribunales que en los noventa resguardaron las prioridades del estado subsidiario por sobre la vida de las personas reconocieron progresivamente que el derecho a la salud y a la vida están relacionados, $\mathrm{y}$ se adhirieron a los postulados del liberalismo igualitario apuntando a garan- 
tizar un mínimo sanitario decente y universal (Zúñiga 2011), acompañando la implementación de la mayor reforma al sistema público de salud introducida a la fecha, la de 2005. En el ámbito de la salud privada el sistema judicial reconoció ciertos abusos de las ISAPREs, aunque ello constituyó un avance limitado, siendo que los costos de la judicialización fueron traspasados por completo a sus afiliados (Atria 2019).

Finalmente, los casos que involucraron temas valóricos han revelado que los tribunales inferiores tienden a ser menos conservadores que los tribunales superiores (Muñoz 2014), y que estos últimos han abandonado la tradicional deferencia hacia el sistema político, oponiéndose a decisiones tomadas por el ejecutivo, como en el caso de la píldora, o a decisiones que el gobierno sí estaba dispuesto a tomar, como en el caso del AUC. El fallo del TC de 2017, que despenaliza el aborto en tres causales, deja entrever que el conservadurismo de los tribunales superiores está sometido a prueba, no sin reticencias del mismo TC, que en 2018 declaró la inconstitucionalidad del reglamento de objeción de consciencia, aprobado para implementar la despenalización.

En esencia, los mayores avances en términos de expansión de los derechos en el Chile post transición no han sido impulsados por los tribunales, sino por la acción colectiva, que supo utilizar la imagen de unos tribunales que ya no reflejaban las exigencias y valores de una sociedad en transformación para instar a sus representantes a tomar la iniciativa política.

\section{REFERENCIAS}

Allard Soto, Raúl, Mônia Clarissa Hennig Leal y Liliana Galdámez Zelada. 2016. "El derecho a la salud y su (des)protección en el estado subsidiario". Estudios Constitucionales, 14 (1): 95-138.

Almond, Gabriel y Sydney Verba. 1963. The civic culture: Political Attitudes and Democracy in Five Nations. Newbury Park: SAGE.

Amenta, Edwin, Neal Caren, Tina Fetner y Michael Young. 2002. "Challengers and States: Toward a Political Sociology of Social Movements". Sociological Views on Political Participation in the 21st Century (10): 47-83.

Andersen, Ellen Ann. 2005. Out of the Closets and Into the Courts: Legal Opportunity Structure and Gay Rights Litigation. Ann Arbor: The University of Michigan Press.

Atria, Fernando. 2019. "Cada vez que el poder de las empresas está en riesgo, los que pierden son los ciudadanos". Entrevista a Radio Futuro, 04-04-2019. Recuperado en: https:/ / www.futuro.cl/2019/04/fernando-atria-abogado-constitucionalista-cada-vez-que-el-poder-de-las-empresas-esta-en-riesgo-los-que-pierden-son-los-ciudadanos/

Barkan, Steven. 2013. "Beware of Lawyers Bearing Gifts, or Why Social Movements Should Be Wary of Litigation". Mobilizing ideas, 4 de febrero de 2013. Recuperado en: https://mobilizingideas.wordpress.com/2013/02/04/beware-of-lawyers-bearinggifts-or-why-social-movements-should-be-wary-of-litigation/

Barnes, Samuel y Kaase, Max. 1979. Political Action: Mass Participation in Five Western Democracies. Beverly Hills: SAGE. 
Boutcher, Steven A. 2010. "Mobilizing in the shadow of the law: Lesbian and gay rights in the aftermath of "Bowers v. Hardwick." Research in Social Movements, Conflict, and Change, Vol. 31: 175-205

Brinks, Daniel. 2012. "The Transformation of the Latin American State-As-Law: State Capacity and the Rule of Law." Revista de Ciencia Politica 32(3): 561-583.

Brinks, Daniel y Varun Gauri. 2014. "The law's majestic equality? The distributive impact of judicializing social and economic rights". Perspectives on Politics 12(2):375-93.

Brown-Nagin, Tomiko. 2005. "Elites, Social Movements and the Law: the Case of Affirmative Action". Columbia Law Review 105(5): 1436-1528.

Casas, Lidia. 2008. La saga de la anticoncepción de emergencia en Chile: avances y desafíos. Serie Documentos Electrónicos N², Programa Género y Equidad, FLACSO Chile.

Coglianese, Cary. 2001. "Social Movements, Law, and Society: The Institutionalization of the Environmental Movement". University of Pennsylvania Law Review 150(1): 85-118.

Contesse, Jorge y Domingo Lovera. 2008. "Acceso a tratamiento médico para personas viviendo con VIH/SIDA: éxitos sin victoria en Chile". Sur. Revista internacional de direitos humanos 5 (8): 150-169, São Paulo.

Dahl, Robert. 1957. "Decision-Making in a Democracy: The Supreme Court as a National Policy-Maker." Journal of Public Law 279(6): 279-295.

Depoorter, Ben. 2013. "The Upside of Losing". Columbia Law Review 113(3): 817-862.

Eisinger, Peter. 1973. "The conditions of protest behavior in American cities". American political Science Review 67(1): 11-28.

Epp, Charles. 1998. The Rights Revolution: Lawyers, Activists, and Supreme Courts in Comparative Perspective, Chicago y Londres: The University of Chicago Press,.

García, José Francisco y Verdugo, Sergio. 2013. Activismo judicial en Chile. ¿Hacia el gobierno de los jueces? Santiago: Libertad y desarrollo.

Gloppen, Siri. 2013. "Social Movement Activism and the Courts". Mobilizing Ideas, 18 de febrero de 2013. Recuperado en: https:/ / mobilizingideas.wordpress.com/2013/02/04/ social-movement-activism-and-the-courts /

Guerra, Felipe. 2017. Los Tribunales Ambientales en la implementación de los derechos indígenas durante la evaluación ambiental de proyectos de inversión en Chile. ocumento de trabajo. Recuperado en: https:/ /observatorio.cl/2768-2/

Handler, Joel F. 1978. Social Movements and the Legal System: A Theory of Law Reform and Social Change. Institute for Research on Poverty Monograph Series. New York: Academic Press.

Hilbink, Lisa. 2007. Judges beyond politics in democracy and dictatorship: Lessons from Chile. New York: Cambridge University Press.

Hilson, Chris. 2002. "New social movements: the role of legal opportunity". Journal of European Public Policy 9(2): 238-255.

Irarrázabal, Ricardo y Sebastián Luengo. 2018. “Aplicación de principios ambientales en el control jurisdiccional del actuar de la Administración". Revista de Derecho Ambiental 6(9): 1-30.

Levi, Margaret. 2006. "Modelando procesos históricos complejos con narrativas analíticas". Revista Uruguaya de Ciencia Política 15(1): 11-29..

McCann, Michael W. 1994. Rights at Work: Pay Equity Reform and the Politics of Legal Mobilization. Chicago: University of Chicago Press.

McCarthy, John D. y Mayer N. Zald. 1977. "Resource Mobilization and Social Movements: A Partial Theory." American Journal of Sociology 82(6): 1212-41.

Meyer, David y Barbara Minkoff. 2004. "Conceptualizing Political Opportunity". Social Forces 82(4): 1457-1492.

Morris, Aldon. 1981. "Black Southern Student Sit-In Movement: An Analysis of Internal Organization". American Sociological Review 46(6): 744-67.

Muñoz, Fernando. 2014. "Morning-After Decisions: Legal Mobilization Against Emergency Contraception in Chile". Michigan Journal of Gender and Law 21(123): 123-175. 
Navarro, Enrique. 2012. “35 años del recurso de protección: notas sobre su alcance y regulación normativa". Estudios constitucionales 10(2): 617-642.

NeJaime, Douglas. 2011. “Winning Through Losing". Iowa Law Review 96: 941-1012.

O’Donnell, Guillermo A. 2001. "Democracy, Law and Comparative Politics". Studies in Comparative International Development 36(1): 7-36.

O'Donnell, Guillermo A. 2010. Democracy, Agency, and the State: Theory with Comparative Intent. New York: Oxford University Press.

Pieterse, Marius. 2008. "Health, Social Movements, and Rights-Based Litigation in South Africa". Journal of Law and Society 35(3): 364-388.

Rosenberg, Alexander. 1991. "Selection and Science: Critical Notice of David Hull's Sciences as a Process". Biology and Philosophy 7: 217-228.

Scheingold, Stuart. 1974. The politics of rights: Lawyers, public policy, and political change. New Haven: Yale University Press.

Sikkink, Kathryn. 2011. The Justice Cascade: How Human Rights Prosecutions are Changing World Politics. New York: W.W. Norton\&Co.

Simmons, Beth A. 2009. Mobilizing for Human Rights: International Law in Domestic Politics. New York: Cambridge University Press.

Smulovitz, Catalina. 2008. “La política por otros medios: Judicialización y movilización legal en la Argentina". Desarrollo económico 48(190/191): 287-305.

Tarrow, Sidney. 1998. Power in Movement: Social Movements and Contentious Politics. New York: Cambridge University Press.

Tata, Neal y Torbjorn Vallinder. 1995. The Global Expansion of Judicial Power. New York: NYU Press.

Vaccario, Anna. 2015. "Il valore del precedente nell'ordinamento giuridico italiano". Tesi del Corso di Teoria dell'interpretazione, Universitá degli Studi di Padova. Recuperado en: https://www.academia.edu/9776813/Il_Valore_del_Precedente_nellOrdinamento_Giuridico_Italiano

Van der Heijden, Hein-Anton. 2006. "Globalization, Environmental Movements, andInternational Political Opportunity Structures". Organization \& Environment 19(1): 28-45

Vanhala, Lisa. 2011. "Social movements lashing back: Law, social change and intra- social movement backlash in Canada". Studies in Law, Politics and Society 54: 113-140.

Verba, Sidney. 1967. "Democratic Participation". The ANNALS of the American Academy of Political and Social Science 373(1): 53-78.

Verba, Sidney, Norman Nye y Jae-on Kim. 1978. Participation and Political Equality: A Seven-Nation Comparison. New York and London: Cambridge University Press.

Verba, Sidney y Robert Nye. 1987. Participation in America. Chicago: University of Chicago Press.

Yamin, Alicia Ely y Siri Gloppen. 2011. Litigating Health Rights: Can Courts Bring More Social Justice to Health? Cambridge: Harvard University Press.

Zemans, Frances Kahn. 1983. "Legal Mobilization: The Neglected Role of the Law in the Political System". The American Political Science Review 77(3): 690-703.

Zúñiga, Alejandra. 2011. "El derecho a la vida y el derecho a la protección de la salud en la constitución: una relación necesaria". Estudios constitucionales 9(1): 37-64.

\section{Entrevistas}

\#2 Alonso Barros, abogado derecho indígena (16-11-2016).

\#5 Colombina Schaeffer, ex subdirectora de proyectos Chile Sustentable (02-09- 2016).

\#8 Diego Lillo, Coordinador de litigios FIMA (08-09-2016).

\#11 Ezio Costa, Director ejecutivo FIMA (03-06-2015)

\#14 Héctor Sánchez, ex Superintendente de ISAPREs (14-12-2016).

\#18 Juan Enrique Jara, líder movimiento pro-vida (10-07-2017).

\#21 Macarena Soler, abogada Geute Conservación Sur (01-09-2016).

\#22 Manuel Inostroza, ex Superintendente de Salud (12-12-2016). 
\#24 Matías Meza-Lopehandía, Profesor de derecho UCV (22-01-2016).

\#28 Pedro Barría, Jefe Unidad de Mediación en Salud, Consejo de Defensa del Estado (28-122016).

\#30 Rolando Jiménez, Representante legal MOVILH (25-08-2016). \#32 Sebastián Pavlovic, ex Superintendente de Salud (07-11-2016).

\#33 Matías Guiloff, Profesor de Derecho Administrativo UDP (27-04-2016).

\#OP1 Reunión de coordinación de movimientos santiagüinos con delegación de líderes indígenas, Centro Costramet, Santiago (23-01-2016).

\#OP3 Trawün en Ruka Walüng, Curarrehe, sector de Trancura (22-02-2016).

Recibido: 6 de diciembre de 2018

Aceptado: 30 de octubre de 2019

Anita Perricone es Doctora en Ciencia Política del Instituto de Ciencia Política de la PUC (2017). A lo largo de su carrera académica se ha enfocado en el estudio de la zona fronteriza entre politica y derecho, la que culminó con la defensa de su tesis doctoral sobre la movilización legal en el Chile post-transición. Además, ha trabajado tópicos de derecho internacional humanitario, de derecho de los pueblos indígenas y movimientos sociales. Correo electrónico: aperricone@uc.cl 